University of Warwick institutional repository: http://go.warwick.ac.uk/wrap This paper is made available online in accordance with publisher policies. Please scroll down to view the document itself. Please refer to the repository record for this item and our policy information available from the repository home page for further information.

To see the final version of this paper please visit the publisher's website. Access to the published version may require a subscription.

Author(s): T. D. Veal and C. F. McConville Article Title: Controlled oxide removal for the preparation of damage-free InAs(110) surfaces

Year of publication: 2000

Link to published version: http://dx.doi.org/10.1063/1.1310211

Publisher statement: None 


\title{
Controlled oxide removal for the preparation of damage-free InAs(110) surfaces
}

\author{
T. D. Veal and C. F. McConville ${ }^{a)}$ \\ Department of Physics, University of Warwick, Coventry, CV4 7AL, United Kingdom
}

(Received 7 April 2000; accepted for publication 18 July 2000)

\begin{abstract}
Controlled oxide removal from InAs(110) surfaces using atomic hydrogen $\left(\mathrm{H}^{*}\right)$ has been achieved by monitoring the contaminant vibrational modes with high resolution electron energy loss spectroscopy (HREELS). The contributing oxide vibrational modes of the partially $\mathrm{H}^{*}$ cleaned surface have been identified. Following hydrocarbon desorption during preliminary annealing at $360^{\circ} \mathrm{C}$, exposure to atomic hydrogen at $400{ }^{\circ} \mathrm{C}$ initially removes the arsenic oxides and indium suboxides; complete indium oxide removal requires significantly higher hydrogen doses. After a total molecular hydrogen dose of $120 \mathrm{~kL}$, a clean, ordered surface, exhibiting a sharp $(1 \times 1)$ pattern, was confirmed by low energy electron diffraction and x-ray photoelectron spectroscopy. Energy dependent HREELS studies of the near-surface electronic structure indicate that no residual electronic damage or dopant passivation results from the cleaning process. () 2000 American Institute of Physics. [S0003-6951(00)05537-6]
\end{abstract}

The preparation of clean, ordered, and stoichiometric III-V semiconductor surfaces is generally problematic without the extensive facilities available with molecular beam epitaxy (MBE). When these are unavailable, the standard method of in situ cleaning of polar InAs surfaces is cycles of low energy ion bombardment and annealing: ${ }^{1}$ however, this has been shown to cause severe structural damage, resulting in the introduction of defects, enhanced carrier concentrations, and reduced mobilities within the outermost $1000 \AA$ of the material. ${ }^{1-3}$ Traditionally, the nonpolar (110) surfaces of III-V materials have been produced by cleaving in vacuum. However, from cleave to cleave large variations in the surface step densities result. These steps have been observed using scanning tunneling microscopy where (111), (112), and (110)-type steps have all been identified on $\operatorname{InAs}(110){ }^{4}$ The consequent modification of the surface, where the reactivity is likely to be dominated by the step density profile, will result in significant nonuniformity in the surface properties with each cleave. This is evident from photoemission studies, where the expected flat-band condition, with no electron accumulation, is rarely achieved for InAs(110). The surface electronic structure is extremely sensitive to the presence of cleave-induced steps, which result in downward band bending and accumulation layer formation. ${ }^{5}$ An alternative surface preparation technique, atomic hydrogen cleaning (AHC), offers an effective way of obtaining clean, undamaged $\operatorname{InAs}(110)$ surfaces with uniform step densities. This method has previously been shown to be an efficient, relatively low temperature technique for obtaining clean, wellordered, damage-free GaAs and InP (001) surfaces. ${ }^{6,7}$

In this letter, the removal of oxide and hydrocarbon contamination from the InAs(110) surface by annealing under a flux of atomic hydrogen $\left(\mathrm{H}^{*}\right)$ is reported. During $\mathrm{AHC}$, the contaminant vibrational modes were identified and monitored by high resolution electron energy loss spectroscopy

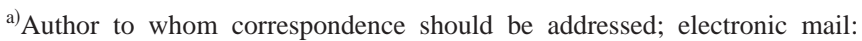
C.F.McConville@warwick.ac.uk
}

(HREELS). The near-surface electronic structure of the clean surface was also studied by HREELS, semiclassical dielectric theory simulations, ${ }^{8}$ and charge profile calculations.

Samples of $S$-doped InAs(110) wafers $\quad(n \sim 4$ $\times 10^{18} \mathrm{~cm}^{-3}$ ) were individually loaded into the ultrahigh vacuum HREELS spectrometer (base pressure $\sim 2$ $\times 10^{-10}$ mbar) without any ex situ treatment. X-ray photoelectron spectroscopy (XPS) and low energy electron diffraction (LEED) were used to monitor the chemical composition and surface ordering, respectively, before, during, and after the cleaning process. Specular HREELS $\left(\theta_{i}=\theta_{s}=45^{\circ}\right)$ was used to study the surface vibrational modes of the contaminants on the as-loaded and partially cleaned surfaces, and to probe the near-surface electronic structure of the clean surface. Molecular hydrogen was thermally cracked by the $\mathrm{W}$ filament of an atomic hydrogen source (EPI Ltd., UK) held at $\sim 2100{ }^{\circ} \mathrm{C}$ (as measured by an infrared pyrometer) with an estimated $\mathrm{H}_{2} \rightarrow \mathrm{H}^{*}$ conversion efficiency of about $6 \%-7 \%$. ${ }^{9}$ Hydrogen doses were measured in terms of molecular hydrogen exposures in kilo-Langmuirs $(\mathrm{kL})\left(1 \mathrm{~kL}=10^{-3}\right.$ Torr s). During AHC, the chamber pressure was typically 3 $\times 10^{-5}$ mbar. Samples were degassed for one hour to $360{ }^{\circ} \mathrm{C}$ prior to $\mathrm{AHC}$, and annealed to $400{ }^{\circ} \mathrm{C}$ during $\mathrm{AHC}$ by a combination of the atomic hydrogen source filament and the sample filament mounted behind. The surface temperature was measured by a chromel-alumel thermocouple in direct contact with the sample.

The as-loaded samples gave a very weak and broad specular elastic peak in HREELS, with an angular spread of $15^{\circ}$ full width at half maximum (FWHM); they exhibited no ordered LEED pattern. Figure 1 shows specular HREEL spectra from an as-loaded sample, after annealing at $360^{\circ} \mathrm{C}$, and following total atomic hydrogen cleaning doses of 15 , 30 , and $60 \mathrm{~kL}$. The as-loaded spectrum [Fig. 1(a)] features a peak at $106 \mathrm{meV}$-almost twice the width of the elastic peak. This is attributed to a combination of $\mathrm{In}-\mathrm{O}$ and the As-O vibrational modes, known to exist at $\sim 87$ and $\sim 118$ meV, respectively. ${ }^{10}$ However, it is apparent that the broad 


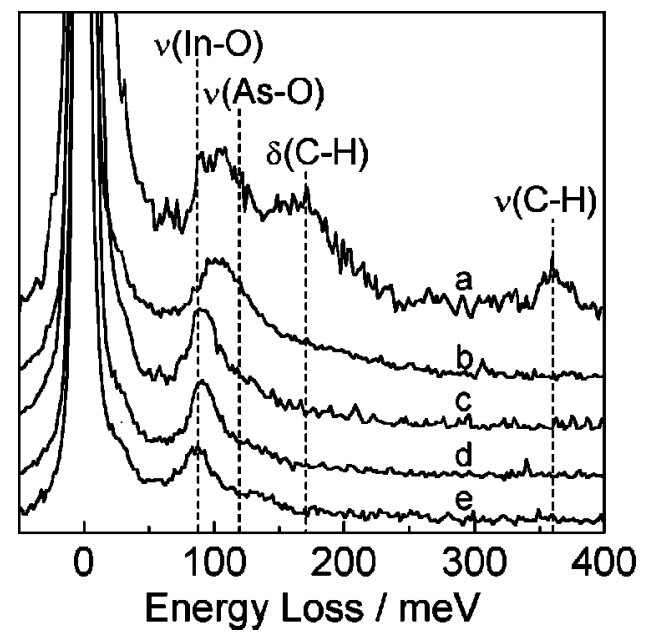

FIG. 1. Specular HREEL spectra, recorded at $15 \mathrm{eV}$, with an instrumental broadening of $16 \mathrm{meV}$ FWHM, from (a) an as-loaded InAs(110) sample; (b) after annealing for one hour at $360{ }^{\circ} \mathrm{C}$; and after total atomic hydrogen cleaning doses of (c) 15, (d) 30, and (e) $60 \mathrm{~kL}$.

peak at $106 \mathrm{meV}$ in both Figs. 1(a) and 1(b) cannot be due solely due to the combination of two symmetric peaks centered at 87 and $118 \mathrm{meV}$. Since the character of the intramolecular bond changes, for example between $\operatorname{In}_{2} \mathrm{O}$ and $\operatorname{In}_{2} \mathrm{O}_{3}$, relatively large frequency shifts can occur. This suggests that the origin of the low loss energy side of this peak is a combination of In-O vibrational modes of several different frequencies in the range 87 to $\sim 100 \mathrm{meV}$. These modes result from the various intramolecular bonds present in the different In oxides likely to exist in the native oxide, including $\mathrm{In}_{2} \mathrm{O}_{3}$ and less thermally stable suboxides such as $\operatorname{In}_{2} \mathrm{O}$. The peak centered at $\sim 170 \mathrm{meV}$, which has been previously assigned incorrectly to the As-O vibration, ${ }^{11}$ is in fact due to the combined hydrocarbon deformation modes, $\delta(\mathrm{C}-\mathrm{H}),{ }^{12}$ while the peak at $360 \mathrm{meV}$ is attributed to the $\mathrm{C}-\mathrm{H}$ stretching mode. The presence of hydrocarbons on the surface was confirmed by the C $1 s$ XPS spectrum. The oxide vibrational modes were identified with the presence of In oxide and $\mathrm{As}_{2} \mathrm{O}_{3}$, by reference to the In $3 d$, As $2 p$ and $3 d$, and $\mathrm{O} 1 s$ XPS spectra. ${ }^{13}$ The In oxide could not be identified by XPS since the different oxides are indistinguishable because of the small chemical shifts of the In $3 d$ lines.

Preannealing at $360{ }^{\circ} \mathrm{C}$ removed the hydrocarbon contaminants, as indicated by the lack of any corresponding vibrational modes in the HREEL spectrum [Fig. 1(b)] and by XPS, although the broad oxide peak at $106 \mathrm{meV}$ remained. This suggests that no oxides are desorbed as a result of annealing at $360^{\circ} \mathrm{C}$. After a $15 \mathrm{~kL}$ dose of $\mathrm{H}^{*}$, a peak at 90 $\mathrm{meV}$ can be observed [Fig. 1(c)], narrower than the initial oxide peak of the annealed surface, but of greater intensity and with a shoulder extending to $\sim 150 \mathrm{meV}$. The change in shape and shift of the oxide peak is due to the removal of most $\mathrm{As}_{2} \mathrm{O}_{3}$ and all the In suboxides, indicating that the peak at $90 \mathrm{meV}$ is mainly composed of $\operatorname{In}_{2} \mathrm{O}_{3}$-related $\mathrm{In}-\mathrm{O}$ vibrations (at $87 \mathrm{meV}$ ), with the high loss energy shoulder due to the residual As-O modes. Desorption of $\mathrm{As}_{2} \mathrm{O}_{3}$ requires a lower $\mathrm{H}^{*}$ dose than $\mathrm{In}_{2} \mathrm{O}_{3}$ removal because of its lower thermal stability, as indicated by the free energies of formation. ${ }^{14}$ The removal of As oxides before Ga oxides has been previously observed during atomic hydrogen cleaning of Downloaded 06 Jul 2009 to 137.205.202.8. Redistribution subjec

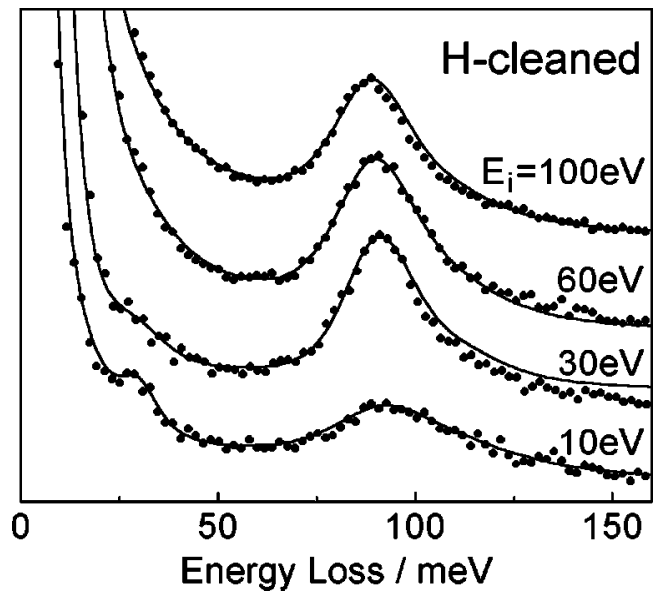

FIG. 2. Experimental specular HREEL spectra, with an instrumental broadening of $10 \mathrm{meV}$ FWHM, recorded at room temperature (dotted lines) and three-layer dielectric theory simulations (solid lines) as a function of incident-electron energy for clean $\operatorname{InAs}(110)\left(n \sim 4 \times 10^{18} \mathrm{~cm}^{-3}\right)$ after a total of $120 \mathrm{~kL}$ of atomic hydrogen cleaning.

GaAs. ${ }^{15,16}$ In the case of $\operatorname{GaAs}(100)$, it has been proposed that the removal of $\mathrm{As}_{2} \mathrm{O}_{3}$ occurs by the formation and desorption of $\mathrm{AsH}_{3}$, while the oxygen generated in this process reacts with surface gallium to form more $\mathrm{Ga}_{2} \mathrm{O}_{3} \cdot{ }^{16} \mathrm{~A}$ similar mechanism is proposed here, with the formation of more $\mathrm{In}_{2} \mathrm{O}_{3}$ explaining the increased intensity in the $\mathrm{In}-\mathrm{O}$ vibrational mode observed in Fig. 1(c).

The HREEL spectrum after a total of $30 \mathrm{~kL}$ of AHC [Fig. 1(d)] is essentially the same as after $15 \mathrm{~kL}$, except that the intensity of the oxide vibration has decreased, suggesting that some $\operatorname{In}_{2} \mathrm{O}_{3}$ has been removed. After a total of $60 \mathrm{~kL}$ of AHC, the oxide peak in the HREEL spectrum [Fig. 1(e)] shifts to $87 \mathrm{meV}$, the position for the In-O vibration. There is no intensity in the As-O vibration region of this spectrum. This corresponds to the removal of $\mathrm{As}_{2} \mathrm{O}_{3}$ to below the detection limit of the technique $\left(\sim 0.1 \%\right.$ of a monolayer $\left.{ }^{10}\right)$, and indicates the presence of $\operatorname{In}_{2} \mathrm{O}_{3}$ as the only surface contaminant. At this stage, the partially clean surface gave a slightly more intense specular elastic peak in HREELS, with an angular spread of $8^{\circ} \mathrm{FWHM}$, and a weak $(1 \times 1)$ LEED pattern with high background intensity. The intensity of all the peaks in the spectra of Fig. 1 decreased as the incident electron energy was increased, with the expected $E_{i}^{-1 / 2}$ dependence, confirming that they are due to vibrational modes and not collective excitations.

A clean surface was obtained after a total of $120 \mathrm{~kL}$ of AHC exhibiting a clear, sharp $(1 \times 1)$ LEED pattern and no residual carbon or oxygen as observed using XPS. Increased surface ordering was also apparent from the more intense specular elastic peak in HREELS with a much reduced angular spread ( $\left.3^{\circ} \mathrm{FWHM}\right)$. HREEL spectra, with varying incident electron energy, are shown in Fig. 2 for the clean surface (dotted lines), along with simulations generated by semiclassical dielectric theory (solid lines). While the clean surface spectra shown do not extend to the loss energies of all the contaminant vibrational modes, it was independently confirmed that their intensities had been reduced to below the detection limit. Instead, two collective excitation features of the clean surface are revealed. In the 10 and $30 \mathrm{eV}$ spectra, a loss feature at $\sim 29 \mathrm{meV}$ assigned to the FuchsAIP license or copyright; see http://apl.aip.org/apl/copyright.jsp 


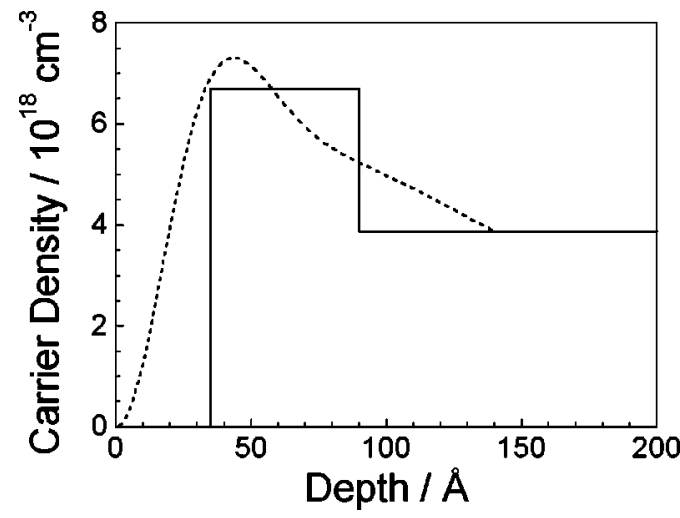

FIG. 3. The MTFA electron density profile for $\operatorname{InAs}(110)$ surfaces $(n \sim 4$ $\times 10^{18} \mathrm{~cm}^{-3}$ ) prepared by atomic hydrogen cleaning (dotted line) and the three-layer histogram profile used in the dielectric theory HREELS simulations (solid line). Note the shape of the profile is characteristic of heavily doped InAs.

Kliewer surface optical phonon can be seen as a shoulder on the elastic peak. The intensity of this feature decays rapidly with increasing incident electron energy, and it is not present in the higher energy spectra. The higher loss energy peak is due to the conduction band surface plasmon excitations, and exhibits a downward dispersion with increasing incident electron energy, from $93 \mathrm{meV}$ in the $10 \mathrm{eV}$ spectrum to 89 $\mathrm{meV}$ at $100 \mathrm{eV}$.

The plasma dielectric function used in the simulations was based on the hydrodynamic model, with the plasma frequencies, plasmon lifetimes, and spatial dispersion coefficients as parameters. ${ }^{2}$ A three-layer model, consisting of a plasmon-free surface "dead" layer, accumulation layer, and a semi-infinite bulk, was used to simulate the experimental spectra across the entire incident electron energy range. The modified Thomas-Fermi approximation (MTFA) with a triangular potential well was used to estimate the layer thicknesses and the plasma parameters (except for the plasmon lifetimes). As we have previously discussed, for the purposes of comparison with histogram profiles derived from dielectric theory simulations of HREEL spectra, deviations between the profile calculated using a triangular potential well and the full classical potential are not significant. ${ }^{17}$ The calculated MTFA carrier density profile is shown in Fig. 3, with a corresponding histogram profile used in the dielectric theory simulations to give the best fit to the experimental spectra. Below the $35 \AA$ dead layer, the $55 \AA$ accumulation layer has a carrier concentration of $6.7 \times 10^{18} \mathrm{~cm}^{-3}$ above a semi-infinite bulk, with a carrier concentration of 3.9 $\times 10^{18} \mathrm{~cm}^{-3}$. This is unchanged from the nominal bulk doping value, indicating that the AHC process does not introduce any detectable dopant passivation. The band bending was estimated to be $115 \mathrm{meV}$, with a triangular potential well width of $140 \AA$, this gives a surface charge density of $6.3 \pm 2.0 \times 10^{11} \mathrm{~cm}^{-2}$. This is in good agreement with the values of $10^{11}-10^{12} \mathrm{~cm}^{-2}$ found on other faces of InAs ${ }^{17,18}$ and on hydrogen terminated $\operatorname{InAs}(110) .{ }^{19}$

The spatial dispersion coefficient for the simulations was calculated using the random phase approximation (RPA), which gives a value of $0.43 \times 10^{6} \mathrm{~ms}^{-1}$ and successfully re- produces the observed plasmon peak dispersion in the experimental spectra. This indicates that AHC does not cause any subsurface damage: the spatial dispersion coefficients for IBA samples are significantly lower than those predicted by the RPA because of additional defect scattering. ${ }^{2}$ When the carrier mobility of the AHC-prepared InAs(110) surfaces is deduced from the plasmon lifetimes used in the simulations, the result is a factor of four higher than for samples from the same wafer subjected to grazing incidence IBA at $500 \mathrm{eV}$ incident energy. This provides further evidence that AHC does not induce the same degree of electronic damage as surfaces subjected to low energy ion bombardment.

In conclusion, it has been demonstrated that clean, well ordered InAs(110) surfaces can be reproducibly obtained by exposure to atomic hydrogen at relatively low anneal temperatures. The removal of hydrocarbon contamination purely by annealing at $360{ }^{\circ} \mathrm{C}$ and controlled reduction of the surface have been achieved, and the vibrational modes of the component oxides identified. In the early stages of AHC $(15-60 \mathrm{~kL})$ at $400{ }^{\circ} \mathrm{C}$, the $\mathrm{As}_{2} \mathrm{O}_{3}$ and In suboxides are shown to be removed. A total molecular hydrogen dose of $120 \mathrm{~kL}$ is required to remove the $\operatorname{In}_{2} \mathrm{O}_{3}$ completely, including that generated during $\mathrm{As}_{2} \mathrm{O}_{3}$ removal. Semiclassical dielectric theory simulations successfully reproduce the experimental clean surface spectra over a wide incident electron energy range. These results indicate that no detectable electronic damage or dopant passivation is introduced in the near-surface region.

One of the authors (T. D. V.) would like to thank the Engineering and Physical Sciences Research Council, U.K. for the provision of a research studentship.

${ }^{1}$ P. H. C. Magnée, S. G. den Hartog, B. J. van Wees, T. M. Klapwijk, W. van de Graaf, and G. Borghs, Appl. Phys. Lett. 67, 3569 (1995).

${ }^{2}$ G. R. Bell, C. F. McConville, and T. S. Jones, Phys. Rev. B 54, 2654 (1996).

${ }^{3}$ G. R. Bell, C. F. McConville, and T. S. Jones, Appl. Surf. Sci. 104/105, 17 (1996).

${ }^{4}$ Y. Liang, W. E. Packard, J. D. Dow, H. Ho, and G. J. Lapeyre, Phys. Rev. B 48, 11942 (1993).

${ }^{5}$ H. S. Karlsson, R. Viselga, and U. O. Karlsson, Surf. Sci. 402-404, 590 (1998).

${ }^{6}$ T. Kikawa, I. Ochiai, and S. Takatani, Surf. Sci. 316, 238 (1994).

${ }^{7}$ E. J. Petit and F. Houzay, J. Vac. Sci. Technol. B 12, 547 (1994).

${ }^{8} \mathrm{Ph}$. Lambin, J. P. Vigneron, and A. A. Lucas, Comput. Phys. Commun. 60, 351 (1990).

${ }^{9}$ EPI Application Note No. 1/96 (1996); A. Sutoh, Y. Okada, and M. Kawabe, Jpn. J. Appl. Phys., Part 2 34, L1379 (1995).

${ }^{10} \mathrm{H}$. Ibach and D. L. Mills, Electron Energy Loss Spectroscopy and Surface Vibrations (Academic, New York, 1982).

${ }^{11}$ G. R. Bell, N. S. Kaijaks, R. J. Dixon, and C. F. McConville, Surf. Sci. 401, 125 (1998)

${ }^{12}$ A. A. Aquino, J. J. Hill, and T. S. Jones, Surf. Sci. 327, 74 (1995).

${ }^{13}$ T. D. Veal and C. F. McConville, Surf. Sci. (to be published).

${ }^{14}$ CRC Handbook of Chemistry and Physics, edited by R. C. Weast (Chemical Rubber, Boca Raton, FL, 1980).

${ }^{15}$ M. Yamada, Y. Ide, and K. Tone, Jpn. J. Appl. Phys., Part 2 31, L1157 (1992).

${ }^{16}$ G. M. Mikhailov, P. V. Bulkin, S. A. Khudobin, A. A. Chumakov, and S. Yu Shapoval, Vacuum 43, 199 (1992).

${ }^{17}$ G. R. Bell, T. S. Jones, and C. F. McConville, Appl. Phys. Lett. 71, 3688 (1997) and references therein.

${ }^{18}$ L. Ö. Olsson, C. B. M. Andersson, M. C. Håkansson, J. Kanski, L. Ilver, and U. O. Karlsson, Phys. Rev. Lett. 76, 3626 (1996).

${ }^{19}$ Y. Chen, J. C. Hermanson, and G. J. Lapeyre, Phys. Rev. B 39, 12682 (1989) 